Annals of Pure and Applied Mathematics

Vol. 13, No. 2, 2017, 223-228

ISSN: 2279-087X (P), 2279-0888(online)

Published on 18 April 2017

www.researchmathsci.org

DOI: http://dx.doi.org/10.22457/apam.v13n2a8

Annals of

Pure and Applied

Mathematics

\title{
Hamiltonian Cycle in Complete Multipartite Graphs
}

\author{
Joseph Varghese Kureethara \\ Department of Mathematics, Christ University \\ Bengaluru - 5620029, Karnataka, India. \\ E-mail: frjoseph@christuniversity.in \\ Received 26 March 2017; accepted 14 April 2017
}

Abstract. This paper deals with the necessary and sufficient conditions for a complete multipartite graph to have a Hamiltonian cycle.

Keywords: Hamiltonian cycle; multipartite graph; tripartite graph

AMS Mathematics Subject Classification (2010): $05 \mathrm{C} 10$

\section{Introduction}

Finding cycles of arbitrary length in connected graphs and complete multipartite graphs has been an interesting type of problem from the elementary to the high order research in the study of graphs. A spanning cycle in a graph is named as the Hamiltonian Cycle after the famous Irish Mathematician Sir William Rowan Hamilton. The problem of finding a Hamiltonian cycle in an arbitrary large graph is NP-complete. Most famous sufficient conditions for the existence of a Hamiltonian cycle in a graph are due to Dirac (1952) and Ore (1960). Billington brought out an extensive survey of cycle decompositions of complete multipartite graphs [1]. A list of the number of Hamiltonian Cycles in various types of graphs is prepared by Weisstein [7]. An extensive survey of the Hamiltonian Problem is due to Gould [2].

Hamiltonian problem is also a distance problem. A link between distance in graph and colouring is explored in [4]. Many results associated with complete multipartite graphs are found in [5] and [6]. Rao Li brings out surprising connection between spectral radius and some Hamiltonian properties of graphs [3]. In this paper we see a necessary and sufficient condition for the existence of Hamiltonian cycle in complete multipartite graphs.

\section{Largest cycle in a complete tripartite graph}

The length of the largest cycle in a complete tripartite of a particular nature is given below.

Lemma 2.1. If $p, q$ and $r$ are the cardinalities of the partite sets of a complete tripartite graph $\mathrm{G}=\mathrm{K}_{\mathrm{p}, \mathrm{q}, \mathrm{r}}$ and if sum of any pair among $\mathrm{p}, \mathrm{q}$ and $\mathrm{r}$ is less than the third number, then the length of the largest cycle in $\mathrm{G}$ is twice the sum of the those pair.

Proof: Since $p, q$ and $r$ are the cardinalities of the partite sets, without loss of generality, let $(p+q)<r$. To get the largest cycle we may try to use the maximum number of vertices 


\section{Joseph Varghese Kureethara}

in G. This maximum is reached only when we consider the sets with $p$ vertices and $q$ vertices together as a single set and the set with $r$ vertices as the second set.

Hence, practically, it works as a bipartite graph with one set containing $p+q$ vertices and the second set with $r$ vertices. Since $(p+q)<r$, the largest cycle possible is of size $2(p+q)$.

\section{Existence of Hamiltonian cycle}

The following lemma describes the coincidence of the largest cycle as the spanning cycle (Hamiltonian Cycle).

Lemma 3.1. Let $p, q$ and $r$ be the cardinalities of the partite sets of a complete tripartite graph $\mathrm{G}=\mathrm{K}_{\mathrm{p}, \mathrm{q}, \mathrm{r}}$. If $\mathrm{p} \leq \mathrm{q} \leq \mathrm{r}$ and if $\mathrm{r}<(\mathrm{p}+\mathrm{q})$, then $\mathrm{G}$ has a Hamiltonian cycle. i.e., $\mathrm{G}$ has a cycle of length $\mathrm{p}+\mathrm{q}+\mathrm{r}$.

Proof: Let P, Q and R be the partite sets of $\mathrm{G}$ with cardinalities $\mathrm{p}, \mathrm{q}$ and $\mathrm{r}$, respectively. Without loss of generality, let us assume that $\mathrm{p}<\mathrm{q}<\mathrm{r}$.

It is given that $r<(p+q)$.

Hence, let $\mathrm{q}=\mathrm{p}+\mathrm{a}$ and $\mathrm{r}=\mathrm{q}+\mathrm{b}$. i.e.,

$$
q=p+a \& r=p+a+b
$$

As $r<(p+q)$, we have $(p+a+b)<(p+p+a)$, using $(1)$.

i.e., $\mathrm{b}<\mathrm{p}$. i.e.,

$$
p-b>0
$$

We can express $\mathrm{p}$ as,

$$
p=(p-b)+b
$$

Using (3), $\mathrm{q}$ and $\mathrm{r}$ can be rewritten as,

$$
q=(p-b)+b+a
$$

and

$$
r=(p-b)+b+a+b
$$

Using the above partitioning of the integers $\mathrm{p}, \mathrm{q}$ and $\mathrm{r}$, sets $\mathrm{P}, \mathrm{Q}$ and $\mathrm{R}$ can be partitioned as follows:

$$
P=P_{(p-b)} \cup P_{b}
$$

where $\left|P_{(p-b)}\right|=p-b$ and $\left|P_{b}\right|=b$.

$$
Q=Q_{(p-b)} \cup Q_{b} \cup Q_{a}
$$

where $\left|Q_{(p-b)}\right|=p-b,\left|Q_{b}\right|=b$ and $\left|Q_{a}\right|=a$.

$$
R=R_{(p-b)} \cup R_{b_{1}} \cup R_{a} \cup R_{b_{2}}
$$

where $\left|R_{(p-b)}\right|=p-b,\left|R_{b_{1}}\right|=b,\left|R_{b_{2}}\right|=b$ and $\left|R_{a}\right|=a$.

Let $V\left(P_{(p-b)}\right)=\left\{u_{1}, u_{2}, \ldots, u_{(p-b)}\right\}$ and $V\left(P_{b}\right)=\left\{u_{(p-b)+1}, u_{(p-b)+2}, \ldots, u_{p}\right\}$.

Let $V\left(Q_{(p-b)}\right)=\left\{v_{1}, v_{2}, \ldots, v_{(p-b)}\right\}, V\left(Q_{b}\right)=\left\{v_{(p-b)+1}, v_{(p-b)+2}, \ldots, v_{p}\right\}$ and

$V\left(Q_{a}\right)=\left\{v_{p+1}, v_{p+2}, \ldots, v_{q}\right\}$. 
Hamilton Cycle in Complete Tripartite Graphs

Let $V\left(R_{(p-b)}\right)=\left\{w_{1}, w_{2}, \ldots, w_{(p-b)}\right\}, V\left(R_{b_{1}}\right)=\left\{w_{(p-b)+1}, w_{(p-b)+2}, \ldots, w_{p}\right\}$ ， $V\left(R_{a}\right)=\left\{w_{p+1}, w_{p+2}, \ldots, w_{q}\right\}$ and $V\left(R_{b_{2}}\right)=\left\{w_{q+1}, w_{q+2}, \ldots, w_{r}\right\}$.

The construction of the cycle is as follows:

We construct a simple path with $u_{1} \in P_{(p-b)} \subset P$ as the initial vertex. Choose $v_{1} \in Q_{(p-b)} \subset Q$ and $w_{1} \in R_{(p-b)} \subset R$ as the next two vertices of the simple path, in that order. After $w_{1}$, we choose $u_{2} \in P_{(p-b)}$. Continuing in this manner, choosing vertices in the increasing order of the suffices of the vertices from $P_{(p-b)}, Q_{(p-b)}$ and $R_{(p-b)}$ in a cyclic manner, we terminate the simple path at $w_{(p-b)} \in R_{(p-b)}$. The length of the simple path thus formed is

$$
3(p-b)-1 \text {. }
$$

In extending the above path, we now consider the sets $Q_{a}$ and $R_{a}$ only. We adjoin the vertex $w_{(p-b)}$ to $v_{p+1} \in Q_{a} \subset Q$. Shuttling between the vertices of $Q_{a}$ and $R_{a}$, we terminate the simple path at $w_{q} \in R_{a}$. Hence, the length of the simple path is thus extended to an additional length of $2 \mathrm{a}$ as,

$$
\left|Q_{a}\right|=\left|R_{a}\right|=a \text {. }
$$

At this level, the vertices of $P_{b}, Q_{b}, R_{b_{1}}$ and $R_{b_{2}}$ are not used in the simple path. As each of these sets has the same cardinality $b$, and since $R_{b_{1}}$ and $R_{b_{2}}$ are subsets of R, we extend the simple path in the following manner.

Adjoin $w_{q}$ to $u_{(p-b)+1} \in P_{b} \subset P$.

Alternating between the $b$ vertices each of $P_{b}$ and $R_{b_{1}}$ we reach the vertex $w_{p} \in R_{b_{1}}$. We adjoin $w_{p}$ to $v_{(p-b)+1} \in Q_{b} \subset Q$.

Shuttling between the $b$ vertices each of $Q_{b}$ and $R_{b_{2}}$ we reach the vertex $w_{r} \in R_{b_{2}}$.

Hence the simple path is now extended with an extra length $4 b$. (11)

As of now, using (9), (10) and (11), the total length of the simple path is

$$
3(p-b)-1+2 a+4 b
$$

Since the path is terminated at $w_{r} \in R_{b_{2}}$, we can complete the cycle by adjoining it to the initial vertex of the path $u_{1} \in P$.

Total length of the cycle thus formed is $3(p-b)-1+2 a+4 b+1$, using (12).i.e., the cycle is of length $3 p+2 a+b$.

However,

$$
3 p+2 a+b=p+p+p+a+a+b, \text { using (1). }
$$

i.e.,

$$
3 p+2 a+b=p+(p+a+b)+(p+a)
$$

i.e.,

$$
3 p+2 a+b=p+q+r
$$


Joseph Varghese Kureethara

Hence, the cycle thus formed is a Hamiltonian Cycle.

\section{Main results}

The discussions in the previous sections lead us to the most important results of this paper. We can estimate the length of the longest cycle in a complete multipartite graph. We start with complete tripartite graph and then we extend the result to complete multipartite graphs.

Theorem 4.1. Let $\mathrm{G}=\mathrm{K}_{p, q, r}$ be a complete tripartite graph such that $\mathrm{p} \leq \mathrm{q} \leq \mathrm{r}$. Then the length of the largest cycle in $\mathrm{G}$ is $\left\{\begin{array}{l}p+q+r, \text { if } r \leq p+q \\ 2(p+q), \text { if } r>p+q\end{array}\right.$.

Proof: Given that the graph $\mathrm{G}$ is complete tripartite. Obviously, length of any cycle will not exceed $p+q+r$, as this gives the total number of vertices in the network. As $p \leq q \leq r$, let us explore the different possibilities in this inequality.

Case 1: $p=q=r$.

In this case, obviously, the length of the largest cycle is $p+q+r$.

Case 2: $p=q<r$.

There can be three different possibilities here, viz.,

$$
r<(p+q), r=(p+q) \text { and }(p+q)<r .
$$

When $r<(p+q)$, we will get a cycle of length $p+q+r$, using Lemma 3.1. If $r=(p+q)$, as $p=q$, we will get without much difficulty, a cycle of length $p+q+r$. When $(p+q)<r$, we will get a cycle of length $2(p+q)$, using Lemma 2.1 .

Case 3: $p<q=r$.

Here as $q>p$, and $q=r, q-p$ and $r$-pvertices will contribute 2( $q$ - $p)$ length of the cycle and the remaining part will contribute $3 p$ to the length of the Hamiltonian cycle. Hence the total length of the Hamiltonian cycle is $2(q-p)+3 p$.

$$
\begin{aligned}
& \text { i.e., }=2 q-2 p+3 p \\
& \text { i.e., }=p+2 q \\
& \text { i.e., }=p+q+r .
\end{aligned}
$$

Case 4: $p<q<r$.

Sub Case 1: $(p+q)<r$.

This is dealt with in Case 2.

Sub Case 2: $(p+q)=r$

This is also dealt with in Case 2.

Sub Case 3: $r<(p+q)$

This case is dealt with in Lemma 3.1.

Hence the proof.

Theorem 4.2. A complete multipartite graph $\mathrm{G}$ of at least three vertices is Hamiltonian if and only if the cardinality of no partite set is larger than sum of the cardinalities of all the other partite sets.

Proof: Suppose that cardinalities one of the partite sets is larger than the sum of the cardinalities of all the other partite sets. As there are only less number of vertices in all 


\section{Hamilton Cycle in Complete Tripartite Graphs}

other partite sets put together, we cannot form a Hamiltonian cycle by pairing elements of the this set and that of the other sets.

Conversely, assume that cardinality of no partite set is larger than sum of the cardinalities of all other sets. We can regroup the partite sets as three sets and by applying Theorem 4.1, form a Hamiltonian cycle.

However, an alternate proof is given below.

Let the complete $\mathrm{m}$-partite graph have the $\mathrm{m}$ partite sets with cardinalities $n_{1} \leq n_{2} \leq \ldots n_{m-1} \leq n_{m}$. It is given that $n_{m} \leq n_{1}+\ldots+n_{m-1}$.

Let $n_{m-2}=p, n_{m-1}=$ qand $n_{m}=r$.

Without loss of generality, let us assume that $p<q<r$.

As $r<p+q$, let $r+a=p+q$. Hence, we have $r=(p-a)+q$.

Let the partite sets with cardinalities $p, q$ and $r$ be $\mathrm{P}$, Q and R respectively.

We now see how a circle of length $p+q+r$ exists among the vertices of the sets $\mathrm{P}$, $\mathrm{Q}$ and $\mathrm{R}$.

Let the vertex sets corresponding to $\mathrm{R}, \mathrm{P}$ and $\mathrm{Q}$ be $\left\{w_{1}, w_{2}, \ldots, w_{r}\right\},\left\{u_{1}, u_{2}, \ldots, u_{p}\right\}$ and $\left\{v_{1}, v_{2}, \ldots, v_{q}\right\}$ respectively. As $r=(p-a)+q$, we can re-label the vertices of $\mathrm{R}, \mathrm{P}$ and $\mathrm{Q}$ as follows:

$$
\begin{aligned}
R & =\left\{w_{1}, w_{2}, \ldots w_{p-a}, w_{p-a+1}, \ldots, w_{(p-a+a)=p}, w_{p+1}, \ldots, w_{(p+q-a)=r}\right\} \\
P & =\left\{u_{1}, u_{2}, \ldots u_{p-a}, u_{p-a+1}, \ldots, u_{(p-a+a)=p}\right\} \text { and } \\
Q & =\left\{v_{(p-a)+1}, \ldots, v_{(p-a)+a=p}, v_{p+1}, \ldots, v_{(p-a)+q=q}\right\} .
\end{aligned}
$$

We get the cycle connecting all these vertices as described below:

We start a simple path from $w_{1}$ to $u_{p-a}$, alternating the vertices of $\mathrm{R}$ and $\mathrm{P}$ in the increasing order of their suffices. From $u_{p-a}$, we extend the path to $w_{(p-a)+1}$. From $w_{(p-a)+1}$ we extend the path to $w_{p+1}$ shuttling among the sets $\mathrm{R}, \mathrm{P}$ and $\mathrm{Q}$ connecting the vertices of them in the increasing order of their suffices. At this stage, all the vertices of $P$ are part of the simple path. Hence, from $w_{p+1}$ we extend the path to $v_{q}$, alternating the vertices of $\mathrm{R}$ and $\mathrm{Q}$ in the increasing order of their suffices.

Now, we have a simple path of length $p+q+r-1$. We now connect $v_{q}$ to $w_{1}$ by an edge to complete a cycle of length $p+q+r$.

It is easy to construct the Hamiltonian cycle using the vertices of other partite sets. As each of the other partite sets has vertices less than that of the set $\mathrm{P}$, as the cycle reaches a vertex of the set $\mathrm{P}$, it can be extended to vertices of other partite sets before it reaches the set R. Hence, in this way a Hamiltonian cycle can be identified in a complete multipartite graph if the cardinality of no partite set is larger than sum of the cardinalities of all the other partite sets.

\section{Conclusion}

Although there are many results coming up frequently regarding the existence of Hamiltonian cycle in various families of graphs, a precise answer is still elusive for general graphs. This article is an attempt to explore the existence of Hamiltonian cycle in 


\section{Joseph Varghese Kureethara}

complete multipartite graphs. The results might be obtained through various other methods. However, a simple algebraic proof is described here.

Acknowledgement. The author would like to express his appreciation to Ms. Roopa Varghese of Indian Institute Science Education and Research, Bhopal, whose internship under the author motivated him to work out the matter discussed in this paper.

\section{REFERENCES}

1. E.J.Billington, Multipartite graph decomposition: cycles and closed trails. Le Matematiche, LIX -Fasc. I-II, (2004) 53-72.

2. R.J.Gould, Recent advances on the Hamiltonian problem: survey III. Graphs and Combinatorics, 30 (2014) 1-46.

3. R.Li, Spectral radius and some Hamiltonian properties of graphs, Annals of Pure and Applied Mathematics, 9 (2015) 125-129.

4. P.T.Marykutty and K.A.Germina, Open distance pattern edge coloring of a graph, Annals of Pure and Applied Mathematics, 6 (2014) 191-198.

5. J.Varghese and A.Antonysamy, On double continuous monotonic decomposition of graphs, Journal of Computer and Mathematical Sciences, 1 (2010) 217-222.

6. J.Varghese and A.Antonysamy, On modified continuous monotonic decomposition of tensor product of graphs, International Journal of Contemporary Mathematical Sciences, 5 (2010) 1609-1614.

7. E.W.Weisstein, Hamiltonian Cycle, MathWorld -- A Wolfram Web Resource. http://mathworld.wolfram.com/HamiltonianCycle.html 